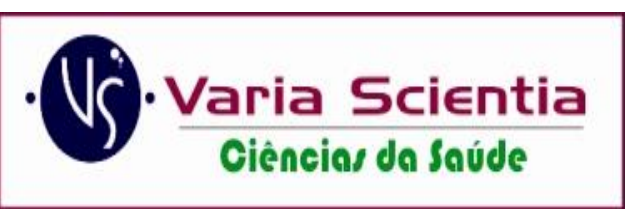

e-ISSN 2446-8118

\title{
EFETIVIDADE DA TERAPIA POR ONDAS DE CHOQUE NO TRATAMENTO DA DOR NA FASCIÍTE PLANTAR: REVISÃO DA LITERATURA
}

\author{
EFFECTIVENESS OF SHOCKWAVES THERAPY IN THE TREATMENT OF PAIN IN \\ PLANTAR FASCIITE: A LITERATURE REVIEW
}

\section{EFICACIA DE LA TERAPIA DE ONDAS DE CHOQUE EN EL TRATAMIENTO DEL DOLOR EN LOS FASCITIS PLANTAR: REVISIÓN DE LA LITERATURA}

\author{
Michelle da Silva Sousa ${ }^{1}$ \\ Carlos Alexandre Batista Metzker ${ }^{2}$
}

\begin{abstract}
RESUMO: Introdução: A fáscia plantar é um tecido fibroso localizado ao longo da planta do pé, que se estende do calcanhar até a base dos metatarsos, com função de sustentação do arco plantar. Uma das principais afecções que ocorrem na fascia plantar é a Fasciíte plantar (FP). Trata-se de inflamação que ocorre devido a um estresse excessivo como impactos, microtraumas repetitivos e processos degenerativos no local de origem da fáscia. Dentre as opções terapêuticas para o tratamento da FP, o método de escolha geralmente é o tratamento conservador, pois, tem se obtido resultados satisfatórios em cerca de $90 \%$ dos pacientes, reduzindo ou abolindo a dor. Nesse cenário, uma das técnicas que vem sendo cada vez mais utilizada para a forma crônica da FP é a terapia por ondas de choque (TOC) extracorpórea, tendo como objetivo promover a cicatrização do tecido inflamado. Objetivo: Verificar e descrever o efeito da terapia por ondas de choque extracorpórea no tratamento da dor em indivíduos com Fasciíte plantar crônica. Método: Foi realizado uma pesquisa nas bases de dados PubMed, Scielo e Lilacs, entre os anos 2016 e 2018 nos idiomas inglês e português. Resultados: Do total de 146 foram incluídos nesse estudo dez artigos que atenderam aos critérios de inclusão e exclusão. Conclusão: A aplicação de TOC parece ser uma alternativa para o manejo da FP crônica com benefícios no controle da dor. Contudo, quando comparada a outras terapias como ultrassom, radiofrequência, laser de baixa intensidade e exercícios domiciliares os resultados foram similares para o desfecho da dor.
\end{abstract}

DESCRITORES: Fasciíte Plantar; Doenças Musculoesqueléticas; Ondas de Choque de Alta Energia; Fisioterapia; Tratamento conservador.

ABSTRACT: Introduction: The plantar fascia is a fibrous tissue located along the sole of the foot, extending from the heel to the base of the metatarsals, supporting the plantar arch. One of the main affections that occur in the plantar fascia is plantar fasciitis (PF). It is inflammation that occurs due to excessive stress such as impacts, repetitive microtraumas and degenerative processes at the origin of the fascia. Among the therapeutic options for the treatment of PF, the method of choice is usually

\footnotetext{
${ }^{1}$ Faculdade de Ensino de Minas Gerais - FACEMG. Discente da Faculdade de Ensino de Minas Gerais - FACEMG.

${ }^{2}$ Faculdade de Ensino de Minas Gerais - FACEMG. Fisioterapeuta, Coordenador e Professor do Curso de Fisioterapia da Faculdade de Ensino de Minas Gerais - FACEMG.

Revista Varia Scientia - Ciências da Saúde, Volume 6 - Número 1 - Primeiro Semestre de 2020.
} 
the conservative treatment, since satisfactory results have been obtained in about $90 \%$ of the patients, reducing or abolishing pain. In this scenario, one of the techniques that is increasingly being used for the chronic form of PF is extracorporeal shockwave therapy (ESWT), aiming to promote the healing of inflamed tissue. Objective: Verify and describe the effect of shockwave therapy in the treatment of pain in individuals with chronic plantar fasciitis. Method: A search was made in the PubMed, Scielo and Lilacs databases between 2016 and 2018 in English and Portuguese. Results: The total of 146, ten articles that met the inclusion and exclusion criteria were included in this study. Conclusion: The application of ESWT seems to be a alternative for the management of chronic PF with benefits in pain control. However, when compared to other therapies such as ultrasound, radiofrequency, low-level laser and home exercises, the results were similar for the outcome of pain.

DESCRIPTORS: Plantar fasciitis; Musculoskeletal Diseases; High-Energy Shock Waves; Physical Therapy Specialty; Conservative treatment.

RESUMEN: Introducción: la fascia plantar es un tejido fibroso ubicado a lo largo de la planta del pie, que se extiende desde el talón hasta la base de los metatarsianos, con la función de sostener el arco plantar. Una de las principales condiciones que ocurren en la fascia plantar es la fascitis plantar (FP). Es una inflamación que ocurre debido a un estrés excesivo, como impactos, microtraumatismos repetitivos y procesos degenerativos en el lugar de origen de la fascia. Entre las opciones terapéuticas para el tratamiento de la FP, el método de elección es generalmente el tratamiento conservador, ya que se han obtenido resultados satisfactorios en aproximadamente el 90\% de los pacientes, lo que reduce o elimina el dolor. En este escenario, una de las técnicas que se ha utilizado cada vez más para la forma crónica de PF es la terapia de onda de choque (TOC) extracorpórea, con el objetivo de promover la curación del tejido inflamado. Objetivo: Verificar y describir el efecto de la terapia de ondas de choque extracorpóreas en el tratamiento del dolor en individuos con fascitis plantar crónica. Método: Se realizó una búsqueda en las bases de datos PubMed, Scielo y Lilacs, entre los años 2016 y 2018 en inglés y portugués. Resultados: De un total de 146, se incluyeron en este estudio diez artículos que cumplieron con los criterios de inclusión y exclusión. Conclusión: La aplicación de TOC parece ser una alternativa para el manejo de la PF crónica con beneficios en el control del dolor. Todavía, en comparación con otras terapias como ultrasonido, radiofrecuencia, láser de bajo nivel y ejercicios caseros, los resultados fueron similares para el resultado del dolor.

DESCRIPTORES: Fascitis Plantar; Enfermedades Musculoesqueléticas; Ondas de Choque de Alta Energía; Fisioterapia; Tratamiento Conservador.

\section{INTRODUÇÃO}

A fáscia plantar é um tecido fibroso localizado ao longo da planta do pé, que se estende do calcanhar até a base dos metatarsos, com função de sustentação do arco plantar. Trata-se de uma estrutura que retém músculos e tendões na planta do pé e dedos, auxiliando no retorno venoso $\mathrm{e}$ reduzindo a compressão das artérias, nervos plantares e digitais ${ }^{1}$.

Uma das principais afecções que ocorrem na fascia plantar é a fasciíte plantar (FP). Trata-se de inflamação que ocorre devido a um estresse excessivo como impactos, microtraumas repetitivos e processos degenerativos no local de origem da fáscia, sendo o diagnóstico essencialmente clínico, tendo como base a história do paciente e o exame físico. A FP pode se tornar crônica, situação que ocorre com frequência em atletas, prejudicando o planejamento desportivo e levando a um longo período de incapacidade $\mathrm{e}^{2,3,4}$.

Comumente, a FP está associada ao excesso de peso, idade média entre 40 a 70 anos, sendo a maior incidência no gênero feminino ${ }^{5}$. Pode ser desencadeada também 
por fatores como sobrecarga na fáscia plantar agregada à fraqueza muscular intrínseca do pé, alterações anatômicas e biomecânicas do arco plantar, uso de calçados inadequados e alterações do posicionamento estático e dinâmico do pé ou até mesmo por discrepância de membros inferiores ${ }^{2,4}$. Sua manifestação é insidiosa, no qual a sintomatologia é caracterizada pela dor ao primeiro apoio matinal e após longos períodos sem apoiar os pés no chão; além de caminhadas prolongadas e longos períodos na posição ortostática ${ }^{3,6}$.

Dentre as opções terapêuticas para o tratamento da FP, o método de escolha geralmente é o tratamento conservador, pois, tem se obtido resultados satisfatórios em cerca de $90 \%$ dos pacientes, reduzindo ou abolindo a dor, restaurando a função mecânica da fáscia plantar e melhorando a marcha ${ }^{1,7,8}$. Nesse cenário, uma das técnicas que vem sendo cada vez mais utilizada para a forma crônica da FP é a terapia por ondas de choque (TOC), que surgiu no Brasil em 1998 como uma nova tecnologia utilizada, tendo como objetivo promover a cicatrização do tecido inflamado ${ }^{9}$. A utilização de ondas de choque aplicadas para afecções do sistema músculo esquelético se iniciou com as máquinas de litotripsia urológica, que logo foram adaptadas para o uso em lesões ortopédicas. Essa adaptação consistiu na introdução de uma tecnologia que permitiu graduar a profundidade e a força com que as ondas de choque penetram no corpo humano $^{10}$.

A ação da TOC é determinada pela penetração das ondas nos tecidos, promovendo estímulo mecânico e induzindo uma série de efeitos biológicos como o aumento na produção de prostaglandinas relacionadas ao processo de reparação tecidual; o aumento da congestão e microcirculação sanguínea local; e o aumento na concentração de óxido nítrico local com alívio da dor ${ }^{11}$. Nesse contexto, foi objetivo desse estudo verificar e descrever o efeito da terapia por ondas de choque extracorpórea no tratamento da dor em indivíduos com Fasciíte plantar crônica.
Revisão de Literatura

\section{MÉTODO}

O presente estudo consistiu de uma revisão integrativa da literatura por meio da busca eletrônica de artigos científicos nacionais e internacionais nas bases de dados PubMed, Scielo e Lilacs, publicados nos idiomas inglês e português a partir dos seguintes descritores: Fasciíte Plantar "plantar fasciitis" / Terapia por ondas de choque "shockwave therapy, / Fisioterapia "Physical Therapy " / Tratamento conservador "conservative treatment" / Dor "Pain".

Foram incluídos artigos originais, disponíveis na íntegra, com período de publicação entre os anos 2016 e 2018, que utilizaram como tratamento conservador para a FP, na forma crônica, a TOC. Foram excluídos, artigos de revisão da literatura, de estudos de caso e que tiveram como proposta principal de tratamento $\mathrm{o}$ ato cirúrgico. Trabalhos que contaram com indivíduos portadores de outras afecções concomitantes no pé e tornozelo, diabetes mellitus, malignidade, neuropatia periférica, implantes metálicos, gravidez, malignidade, uso de anticoagulante e infecções sistêmicas foram também excluídos. Para a seleção dos artigos científicos foram organizados procedimentos por etapas: na primeira etapa foram avaliados e selecionados pela leitura dos títulos e resumos; na segunda etapa os estudos selecionados foram então acessados em sua versão completa para leitura na íntegra. Dois avaliadores independentes analisaram todos os estudos encontrados.

\section{RESULTADOS E DISCUSSÃO}

A partir da revisão de literatura foram encontrados 146 artigos nas bases de dados pesquisadas, sendo excluídos 136 estudos por não atenderem ao objetivo da presente pesquisa e aos critérios adotados. Portanto, a revisão foi composta por dez artigos publicados em periódicos indexados. Os estudos utilizados apresentaram efeitos positivos da TOC em pacientes com FP, com 
ênfase em diminuição da dor e qualidade de artigos incluídos no estudo: vida. A tabela 1 apresenta uma síntese dos

Tabela 1 - Descrição dos artigos analisados, com identificação dos autores, títulos, ano de publicação, tipo do estudo e principais achados.

\begin{tabular}{|c|c|c|c|c|}
\hline Autores & Título & Ano & Tipo estudo & Principais achados \\
\hline $\begin{array}{l}\text { Bicer } \\
\text { et }^{12}\end{array}$ & $\begin{array}{l}\text { Assessment of the Efficacy of } \\
\text { Extracorporeal Shockwave Therapy for } \\
\text { Plantar Fasciitis with Magnetic Resonance } \\
\text { Imaging Findings. }\end{array}$ & 2018 & $\begin{array}{l}\text { Estudo Quasi } \\
\text { experimental }\end{array}$ & $\begin{array}{l}\text { TOC reduziu dor pela } \\
\text { manhã }(\mathrm{p}<0,0001) \\
\text { durante atividades } \\
(\mathrm{p}<0,0001) ; \text { e em } \\
\text { repouso }(\mathrm{p}<0,008)\end{array}$ \\
\hline $\begin{array}{l}\text { Hocaoglu } \\
\text { et al. }{ }^{.4}\end{array}$ & $\begin{array}{l}\text { Comparative Effectiveness of Radial } \\
\text { Extracorporeal Shockwave Therapy and } \\
\text { Ultrasound-Guided Local Corticosteroid } \\
\text { Injection Treatment for Plantar Fasciitis. }\end{array}$ & 2017 & ECA & $\begin{array}{l}\text { TOC > injeção de } \\
\text { corticosteróides para } \\
\text { melhora da dor. }\end{array}$ \\
\hline $\begin{array}{l}\text { Eslamia } \\
\text { et al. } .^{15}\end{array}$ & $\begin{array}{l}\text { Extra Corporeal Shock Wave Therapy } \\
\text { Versus Local Corticosteroid Injection in } \\
\text { the Treatment of Chronic Plantar Fasciitis, } \\
\text { a Single Blinded Randomized Clinical } \\
\text { Trial. }\end{array}$ & 2016 & $\mathrm{ECA}$ & $\begin{array}{c}\text { TOC = injeção de } \\
\text { corticosteróides para } \\
\text { melhora considerável da } \\
\text { dor. }\end{array}$ \\
\hline $\begin{array}{l}\text { Ozan } \\
\text { et al. }\end{array}$ & $\begin{array}{l}\text { Radiofrequency Thermal Lesioning and } \\
\text { Extracorporeal Shockwave Therapy: A } \\
\text { Comparison of Two Methods in the } \\
\text { Treatment of Plantar Fasciitis. }\end{array}$ & 2016 & $\begin{array}{l}\text { Estudo Quasi } \\
\text { experimental }\end{array}$ & $\begin{array}{l}\text { TOC }=\text { radiofrequência } \\
\text { para melhora da } \\
\text { intensidade da dor. }\end{array}$ \\
\hline $\begin{array}{l}\text { Roca, } \\
\text { Mendonza } \\
\text { e Roca }{ }^{17} \\
\end{array}$ & $\begin{array}{l}\text { Comparison of extracorporeal shock wave } \\
\text { therapy with botulinum toxin type } \mathrm{A} \text { in the } \\
\text { treatment of plantar fasciitis. }\end{array}$ & 2016 & $\begin{array}{l}\text { Estudo Quasi } \\
\text { experimental }\end{array}$ & $\begin{array}{l}\text { TOC > toxina botulínica } \\
\text { para melhora da dor. }\end{array}$ \\
\hline $\begin{array}{l}\text { Maki } \\
\text { et } a . l^{18}\end{array}$ & $\begin{array}{l}\text { Magnetic resonance imaging findings of } \\
\text { chronic plantar fasciitis before and after } \\
\text { extracorporeal shock wave therapy. }\end{array}$ & 2017 & $\begin{array}{l}\text { Estudo Quasi } \\
\text { experimental }\end{array}$ & $\begin{array}{l}\text { Melhora da dor até seis } \\
\text { meses após a TOC } \\
(\mathrm{p}<0,05)\end{array}$ \\
\hline $\begin{array}{l}\text { Ulusoy, } \\
\text { Cerrahogl } \\
\text { u e } \\
\text { Orguc. }{ }^{19}\end{array}$ & $\begin{array}{l}\text { Magnetic Resonance Imaging and Clinical } \\
\text { Outcomes of Laser Therapy, Ultrasound } \\
\text { Therapy, and Extracorporeal Shock Wave } \\
\text { Therapy for Treatment of Plantar Fasciitis: } \\
\text { A randomized Controlled Trial. }\end{array}$ & 2017 & ECA & $\begin{array}{l}\text { TOC e LLLT > terapia } \\
\text { por US na melhora da } \\
\text { dor. }\end{array}$ \\
\hline $\begin{array}{l}\text { Akinoglu } \\
\text { e Kose }^{20}\end{array}$ & $\begin{array}{l}\text { A comparison of the acute effects of radial } \\
\text { extracorporeal shockwave therapy, } \\
\text { ultrasound therapy, and exercise therapy in } \\
\text { plantar fasciitis. }\end{array}$ & 2018 & ECA & $\begin{array}{l}\text { TOC }=\text { US e terapia de } \\
\text { exercícios em domicílio } \\
\text { para melhora da dor. }\end{array}$ \\
\hline $\begin{array}{l}\text { Akinoglu } \\
\text { et al. }\end{array}$ & $\begin{array}{l}\text { Comparison of the Acute Effect of Radial } \\
\text { Shock Wave Therapy and Ultrasound } \\
\text { Therapy in the Treatment of Plantar } \\
\text { Fasciitis: A Randomized Controlled Study. }\end{array}$ & 2017 & ECA & $\begin{array}{c}\text { TOC }=\text { US para melhora } \\
\text { da dor. }\end{array}$ \\
\hline $\begin{array}{l}\text { Yin } \\
\text { et al. }\end{array}$ & $\begin{array}{l}\text { A new and accurate predictive model for } \\
\text { the efficacy of extracorporeal shock wave } \\
\text { therapy in managing patients with chronic }\end{array}$ & 2017 & ECA & $\begin{array}{l}\text { TOC potencial para a } \\
\text { redução da dor. }\end{array}$ \\
\hline
\end{tabular}

Legenda: TOC: Terapia por Ondas de Choque, ECA: Estudo controlado Aleatorizado, US:Ultrassom, LLLT: terapia a laser de baixa intensidade.

Fonte: Dados coletados pelos autores, 2018.

Para determinar a eficácia da TOC, Bicer et $a l^{12}$ utilizaram achados de ressonância magnética (RM) em um estudo envolvendo 30 pacientes de ambos os sexos, com faixa etária de 20 a 60 anos, que apresentavam sintomas de FP por pelo menos 6 semanas. Os pacientes foram avaliados com
Escala Visual Analógica (EVA) para determinar o nível de dor pela manhã, durante atividades e em repouso e como instrumento clínico para avaliação funcional do pé e tornozelo usaram o questionário American Orthopaedic Foot and Tornozelo Society (AOFAS), instrumento adaptado à população 
brasileira devidamente validado, que consiste em uma escala que varia de 0 a 100 pontos, em que uma pontuação maior corresponde a uma melhor condição funcional. ${ }^{13}$ Ao final do estudo concluíram que 21 dos 30 pacientes, no qual foram submetidos à TOC e que permaneciam em pé por mais de cinco horas por dia, obtiveram diminuição significativa da dor pós tratamento pela manhã $(\mathrm{p}<0,0001)$; durante atividades $(\mathrm{p}<0,0001)$; e em repouso ( $\mathrm{p}<0,008$ ), o que foi demonstrado nos achados da RM em comparação ao valor pré tratamento.

A terapia por ondas de choque é uma modalidade de tratamento conservador de fácil aplicação e segura, sendo de baixo custo e com taxas de complicações não significativas. Além da diminuição da dor, Bicer et al observaram também melhora do espessamento da fáscia plantar utilizando achados em RM. Os radiologistas realizaram as medições usando um software, contudo, não realizarem um estudo de repetibilidade para garantir a pontuação das imagens.

Do mesmo modo, Hocaoglu et al. ${ }^{14}$ compararam os efeitos clínicos a longo prazo da TOC com a injeção de corticosteroide. Foi realizado um estudo randomizado com 72 pacientes, de ambos os sexos, com faixa etária de 18 a 65 anos, contando com pacientes diagnosticados com fasciíte plantar e refratariedade ao tratamento conservador por mais de seis meses. Amostra dividida em dois grupos compostos com 36 pacientes, onde o grupo um (G1) foi tratado pela TOC, três vezes por semana, pelo mesmo fisioterapeuta; e o segundo grupo $(\mathrm{G} 2)$ recebeu $1 \mathrm{ml}$ de betametasona sódica acrescido de $0,5 \mathrm{ml}$ de prilocaína por injeção de dose única. Ambos os grupos orientados a evitar longos períodos de permanência na posição de pé e atividades extenuantes provocadoras de dor; além de não realizarem exercícios durante uma semana após o tratamento. Ao final do estudo foram observadas melhoras significativas em ambos os grupos, sendo modalidades eficazes no tratamento de fasciíte plantar. No entanto, a TOC mostrou-se superior à terapia com injeção de corticosteroides, pois os resultados apontaram que a TOC proporcionou ação de redução de dor a longo prazo. Nesse estudo os
Revisão de Literatura autores utilizaram como método de avaliação no início, em um, três e seis meses após o tratamento a EVA, índice de funcionalidade do pé (FFI) e medida ultrassonográfica.

Semelhantemente, Eslamia et al. ${ }^{15}$ comprovaram, em um ensaio clínico randomizado, no qual avaliaram a eficácia da TOC e da injeção corticosteroide que, ambos os tratamentos resultam em melhora considerável da dor, embora não tenham sido encontradas diferenças estatisticamente significativas intergrupos. $\mathrm{O}$ que difere é que a injeção de corticosteroides requer um período de descanso após a administração, enquanto a TOC tem benefícios de não imobilização e retorno precoce ao trabalho. Embora a dor ocorra durante a sessão de terapia em ambos os tratamentos, a dor durante a injeção é maior do que a dor na sessão da TOC

Em um estudo randomizado, prospectivo, no qual foram incluídos 56 pacientes de ambos os sexos, com queixas de dor por mais de seis meses Ozan et al. ${ }^{16}$ dividiram a amostra em dois grupos, sendo G1 com 40 pacientes submetidos à TOC, e o G2 com 16 pacientes submetidos à radiofrequência. Como método de avaliação utilizaram a EVA no início, um, três e seis meses pós tratamento. A TOC foi aplicada uma vez por semana, durante 4 semanas, e a radiofrequência foi utilizada durante uma única sessão, ambos por um único técnico. Os autores concluíram que ambos os tratamentos diminuíram a intensidade da dor, aumentando a capacidade de caminhar dos pacientes; além de serem técnicas seguras e eficazes em pacientes com FP crônica.

Tanto o estudo de Hocaoglu et al. ${ }^{14}$ quanto o de Ozan et. al. ${ }^{16}$ compararam os efeitos da TOC a outra terapia: injeção de corticosteroide e radiofrequência térmicas lesionante. Entretanto, Ozan et. al. ${ }^{16}$ submeteram sua amostra a TOC uma vez por semana, o que levou a utilizar a frequência de $15 \mathrm{~Hz}$, já Hocaoglu et al ${ }^{14}$ submeteram sua amostra a terapia três vezes por semana, tendo utilizado a frequência de $10 \mathrm{~Hz}$. Em ambos os estudos houve o cuidado de ser um único técnico a aplicar as terapias, evitando que haja diferença de métodos. 
Roca, Mendonza e Roca ${ }^{17}$ realizaram um estudo prospectivo e randomizado em 72 pacientes de ambos os sexos no qual não responderam à terapia inicial com cinesioterapia e eletroterapia. Participaram do estudo pacientes com faixa etária superior a 15 anos, diagnosticados com FP, com sintomas de pelo menos seis meses. Os participantes foram distribuídos aleatoriamente em dois grupos iguais com 40 indivíduos, sendo que o G1 realizou a TOC no ato da inscrição, um e dois meses depois; e o G2 recebeu uma injeção com toxina botulínica diluída em $1 \mathrm{ml}$ de solução salina normal, também no ato da inscrição, um e dois meses depois. Ambos os grupos utilizaram a EVA para avaliação da dor e orientados a realizarem diariamente exercícios de alongamentos do músculo da panturrilha e arco do pé. Diante de inúmeras variáveis no qual poderiam interferir no resultado final, os autores concluíram que o menor peso e o uso da TOC foram associados à melhora da dor quando comparada à toxina botulínica.

O estudo por Roca, Mendonza e Roca $^{17}$ que utilizou TOC versus toxina botulínica, diferenciou dos demais devido à utilização, em ambos os grupos, de exercícios diários de alongamentos dos músculos da panturrilha e arco plantar, que foram acrescidos ao plano de tratamento. Além disso, utilizou um intervalo maior de tempo com aplicação de apenas uma sessão mensal, diferenciando-se demais estudos que tiveram frequência semanal (uma vez por semana) ${ }^{12,14-}$ 22.

Em um estudo comparativo entre os achados em imagens de RM, antes e após o tratamento da fasciíte plantar com TOC, que incluíram 23 pés (10 homens e 13 mulheres com faixa etária de 16 a 81 anos), com sintomas em média de quatro a 30 meses de duração, Maki et. al. ${ }^{18}$ concluíram que houve melhora da dor seis meses após a TOC $(p<0,05)$, sendo que não houve diferença significativa entre homens e mulheres. Assim, dos 23 pés tratados, 19 apresentaram melhora dos sintomas como também ocorreu nos achados de RM ( $\mathrm{p}<0,01)$. Como método de avaliação foram utilizados a escala de
Revisão de Literatura

tornozelo-retropé e a EVA, antes e seis meses pós-tratamento, com um acompanhamento de seis meses.

$O$ estudo de Maki et al. ${ }^{18}$ confirma benefícios da TOC ao comparar os resultados do tratamento com os achados de RM após a TOC. Nesse estudo, a melhora da dor foi linear a melhora da condição da fáscia plantar, situação que leva a acreditar que a inflamação em torno da FP é melhorada pela ação da estimulação da cicatrização dos tecidos moles, pela remoção de restos inflamatórios e a promoção da neovascularização da TOC contribuindo para uma melhora, além da diminuição da dor.

Em um ensaio clínico randomizado, prospectivo e comparativo entre eficácia da terapia a laser de baixa intensidade (LLLT), ultrassom terapêutico (US) e a TOC, usando ressonância magnética em pacientes com diagnóstico de fasciíte plantar crônica, Ulusoy, Cerrahoglu e Orguc ${ }^{19}$ concluíram que o tratamento com LLLT e TOC apresentaram resultados semelhantes $(p>0,05)$ e ambos foram mais eficazes do que a terapia por US na melhora da dor $(p>0,012)$. Nesse estudo os autores incluíram 60 pacientes com sintomas de calcanhar doloroso por mais de seis meses, sem resposta ao tratamento conservador, sendo os grupos divididos em três: grupo (G1) que foi submetido a 15 sessões de LLLT, grupo (G2) com 15 sessões de US e o grupo (G3) com três sessões de TOC. Sendo avaliados a funcionalidade (AOFAS) limitações, sensibilidade e dor (EVA).

Similarmente, Akinoglu e Kose ${ }^{20}$ compararam em um estudo randomizado, os efeitos da TOC, US e a terapia de exercícios em domicílio para melhora da dor, fadiga e desempenho em 54 mulheres, com idade média de 50 anos, com diagnóstico de fasciíte plantar a mais de três meses. As participantes foram divididas em três grupos com 18 pacientes cada, no qual G1 realizou exercícios com TOC aplicada em três sessões, uma vez por semana; G2 submeteu-se a exercícios e US com um total de sete sessões em dois dias na semana; e o G3 que realizou somente exercícios constituídos de alongamentos do tendão do calcâneo, músculos gastrocnêmio e fáscia plantar 10 x 30 segundos durante 
quatro semanas e duas vezes ao dia. A mensuração da dor se deu pela EVA e ao final concluíram não haver diferenças entre os grupos, tanto na melhora da dor, quanto na fadiga e desempenho ( $p>0,05)$, sendo as três técnicas efetivas para $o$ tratamento conservador da FP.

Da mesma forma em um ensaio clínico randomizado, prospectivo e controlado, em uma amostra com 54 pacientes do sexo feminino, com faixa etária entre 39 a 59 anos de idade, comparando a eficácia da terapia por ondas de choque (TOC) e ultrassom terapêutico (US) em pacientes com diagnóstico de FP avaliados antes e quatro semanas pós tratamento, Akinoglu et $a l^{21}$ concluíram que o tratamento com US e TOC apresentaram diminuição dos sintomas de forma semelhante $(p>0,05)$ sendo eficazes na melhora da dor. Para avaliação os autores utilizaram a EVA.

Em um estudo realizado por Yin et $a l^{22}$ com o objetivo de estabelecer um modelo preditivo de TOC no tratamento de pacientes com FP crônica, os autores concluíram que os parâmetros de 2.400 de impulsos, com densidade de fluxo de energia de $0,2 \mathrm{mj} / \mathrm{mm}^{2}$ $0,4 \mathrm{ml} / \mathrm{mm}^{2} 0,6 \mathrm{ml}$ com 8 a 17 impulsos por segundo têm o potencial de redução da dor em pacientes com FP crônica.

Nessa revisão, a idade dos pacientes foi de 15 a 81 anos e o tamanho das amostras variou de 23 a 80 indivíduos. No que se refere ao período da intervenção houve variação entre os estudos, sendo que o maior foi observado no trabalho de Hocaoglu et al. ${ }^{14}$, no qual submeteu os pacientes em três sessões da TOC por semana por um período de seis meses; seguido pelos estudos de Eslamia et $a l .{ }^{15}$, que realizou cinco sessões de TOC em um intervalo de três dias; e de Ozan et al. ${ }^{16}$, que utilizou a TOC uma vez por semana durante um mês. Não foi evidenciado períodos de intervenção inferior a três semanas, mas houve similaridade entre os demais estudos que aplicaram a TOC em uma sessão durante três semanas, não chegando a um mês de tratamento ${ }^{12,17-, 22}$.

Em relação a frequência utilizada houve variação, sendo de $4 \mathrm{~Hz}^{17}, 15 \mathrm{~Hz}^{12,16} \mathrm{e}$ com vários estudos com $10 \mathrm{~Hz}^{14,15,18-, 22}$.
Revisão de Literatura

Quanto à duração, os pacientes foram submetidos à TOC por 15 minutos. Em nove dos dez estudos, a intervenção para tratamento da dor na FP com utilização da TOC mostrou resultados significativos ${ }^{12,14-22}$. Com relação aos instrumentos para avaliação quantitativa da dor, todos os estudos utilizaram a EVA $^{12,14-22}$. Consiste em uma linha de dez centímetros, que possui em geral, as frases "ausência de dor e dor insuportável", apresentada em uma folha em branco, numerada de zero (ausência), a dez (dor insuportável). Idosos e crianças, às vezes, sentem dificuldades em utilizá-la devido à abstração necessária para sua compreensão. Para essa população específica, existem instrumentos adequados que usam outros recursos visuais como desenhos representando expressões faciais. Os benefícios da utilização da EVA consistem em acompanhar os resultados do tratamento proposto $^{23}$.

\section{CONCLUSÃO}

Ao final do estudo concluiu-se que a aplicação de TOC parece ser uma alternativa para o tratamento da FP crônica com benefícios no controle da dor. Contudo, quando comparada a outras terapias como ultrassom, radiofrequência, laser de baixa intensidade e exercícios domiciliares os resultados foram similares para o desfecho da dor. Além do controle da dor, a TOC pode produzir resultados positivos em relação à função. Mais estudos para explorar essa hipótese são recomendados.

\section{REFERÊNCIAS}

1. Androsoni R, Netto AA, Macedo RR, Fasolin RP, Boni G, Moreira RFG, Tratamento da fasceíte plantar crônica pela terapia de ondas de choque, avaliação morfológica ultrassonográfica e funcional. Rev. Bras. Ortop. 2013;48(6): 538-44.

2. Ferreira RC, Talalgias. Fascite Plantar. Rev. Bras. Ortop. 2014;49(3):213-17. 
3. Metzker CAB, A Fricção Transversa Profunda no Tratamento da Fasceíte Plantar Crônica. Rev. Saúde e Biol. 2012;7(3):12027.

4. Pontin JCB, Costa TR, Chamlian TR, Tratamento fisioterapêutico da fasciíte plantar. Acta Fisiatra. 2014;21(3):147-51.

5. Nahin NC, Prevalence and Pharmaceutical Treatment of Plantar Fasciitis in United States Adults. Official journal of the American Pain Society. 2018; 19(8):885-96

6. Charlotte NC, Plantar Fasciitis: Diagnosis and Conservative Management Miller Orthopaedic Clinic. 2007;5(2):109-17

7. Kertzman P, Lenza M, Penelli A, Ejnisman B, Shockwave treatment for musculoskeletal diseases and bone consolidation: qualitative analysis of the literature Rev. bras. ortop. 2015;50(1):194-99

8. Metzker $\mathrm{CAB}$, Efeitos de um protocolo fisioterapêutico no tratamento da fasceíte plantar: Relato de caso. Rev Ciências Saúde. 2018;3(2):11-17

9. Silva DR, Zang GC, Vidmar MF, Pimentel GL, Terapia por Ondas de Choque Extracorpórea e Laser na redução da dor de indivíduos com fasciíte plantar: rev. sistemática ConScientiae Saúde. 2016;15(4):671-78.

10. Kertzman PF, Duarte JÁ, Terapia por ondas de choque: Uma nova opção para o tratamento de tendinopatias crônicas. Rev Bras Med. 2011;68(1):24-8.

11. Hofmann A, Ritz U, Hessmann MH, Alini M, Rommens PM, Rompe JD, Extracorporeal shock wave-mediated changes in proliferation, differentiation, and gene expression of human osteoblasts. J Trauma. 2008;65(6):1402-10.

12. Bicer M, Hocaoglu E, Aksoy S, Iinci E, Aktas I, Assessment of the Efficacy of
Revisão de Literatura

Extracorporeal Shockwave Therapy for Plantar Fasciitis with Magnetic Resonance Imaging Findings. Journal of the American Podiatric Medical Association. 2018;108(2):100-05.

13. Rodrigues RC, Masiero D, Mizusaki JM, Imoto AM, Peccin MS, Cohen $M$ et al. Tradução, adaptação cultural e validação do "American Orthopaedic Foot and Ankle Society (AOFAS) Ankle-Hindfoot Scale". Acta Ortop Bras. 2008;16(2):107-11.

14. Hocaoglu S, Vurdem EU, Cebicci MA, Sutbeyaz ST, Guldeste Z, Yunsuroglu SG, Comparative Effectiveness of Radial Extracorporeal Shockwave Therapy and Ultrasound-Guided Local Corticosteroid Injection Treatment for Plantar Fasciitis. Journal of the American Podiatric Medical Association. 2017;107(3):192-99.

15. Eslamian F, Shakouri SK, Jahanjoo F, Hajialiloo $\mathrm{M}$ and Notghi F. Extra Corporeal Shock Wave Therapy Versus Local Corticosteroid Injection in the Treatment of Chronic Plantar Fasciitis, a Single Blinded Randomized Clinical Trial. Pain Medicine. 2016;1(10):113-17

16. Ozan F, Koyuncu S, Gurbuz K, Oncel ES, Altay T. Radiofrequency Thermal Lesioning and Extracorporeal Shockwave Therapy: a Comparison of Two Methods in the Treatment of Plantar Fasciitis. Foot \& Ankle Specialist. 2016:20(2):1-6

17. Roca B, Mendoza MA, Roca M. Comparison of extracorporeal shock wave therapy with botulinum toxin type $\mathrm{A}$ in the treatment of plantar fasciitis. Disability and Rehabilitation. 2016;2114-21

18. Maki M, Ikomaa K, Kidoa M, Haraa Y, Sawadab K, Ohashib S, Kuboa T. Magnetic resonance imaging findings of chronic plantar fasciitis before and after extracorporeal shock wave therapy. The Foot, 2017;33:25-8

19. Ulusoy A, Cerrahoglu L, Orguc S. Magnetic Resonance Imaging and Clinical 
Outcomes of Laser Therapy, Ultrasound Therapy, and Extracorporeal Shock Wave Therapy for Treatment of Plantar Fasciitis: A randomized Controlled Trial. The Journal of Foot \& Ankle Surgery. 2017;56: 762-67

20. Akinoglu B, Kose N. A comparison of the acute effects of radial extracorporeal shockwave therapy, ultrasound therapy, and exercise therapy in plantar fasciitis. Journal of Exercise Rehabilitation. 2018:14(2):306-12

21. Akinoglu B, Kose N, Kirdi N, Yakut Y. Comparison of the Acute Effect of Radial Shock Wave Therapy and Ultrasound Therapy in the Treatment of Plantar Fasciitis: A Randomized Controlled Study. Pain Medicine. 2017;18:2443-452

22. Yin M, Chen N, Huang Q, Marla AS, Ma $\mathrm{J}$, Ye J, Mo W. A new and accurate predictive model for the efficacy of extracorporeal shock wave therapy in managing patients with chronic plantar fasciitis. Archives of Physical Medicine and Rehabilitation. 2017;10:1016.

23. Martinez JE, Grassi DC, Marques LG, Análise da aplicabilidade de três instrumentos de avaliação de dor em distintas unidades de atendimento: ambulatório, enfermaria e urgência. Rev Bras Reumatol. Recebido em: 14.07.2020. 2011;51(4):299-308 Aprovado em: 20.07.2020. 